\title{
Evolution of the Primordial Axial Charge across Cosmic Times
}

\author{
A. Boyarsky, ${ }^{1}$ V. Cheianov, ${ }^{1}$ O. Ruchayskiy $\odot,{ }^{2}$ and O. Sobol $\circledast^{3,4, *}$ \\ ${ }^{1}$ Instituut-Lorentz for Theoretical Physics, Universiteit Leiden, Niels Bohrweg 2, 2333 CA Leiden, Netherlands \\ ${ }^{2}$ Niels Bohr Institute, University of Copenhagen, Blegdamsvej 17, DK-2100 Copenhagen, Denmark \\ ${ }^{3}$ Institute of Physics, Laboratory for Particle Physics and Cosmology, École Polytechnique Fédérale de Lausanne, \\ CH-1015 Lausanne, Switzerland \\ ${ }^{4}$ Physics Faculty, Taras Shevchenko National University of Kyiv, 64/13, Volodymyrska Str., 01601 Kyiv, Ukraine
}

(Received 1 August 2020; revised 16 September 2020; accepted 1 December 2020; published 11 January 2021)

\begin{abstract}
We investigate collisional decay of the axial charge in an electron-photon plasma at temperatures $10 \mathrm{MeV}-100 \mathrm{GeV}$. We demonstrate that the decay rate of the axial charge is first order in the fine-structure constant $\Gamma_{\text {flip }} \propto \alpha m_{e}^{2} / T$ and thus orders of magnitude greater than the naive estimate which has been in use for decades. This counterintuitive result arises through infrared divergences regularized at high temperature by environmental effects. The decay of axial charge plays an important role in the problems of leptogenesis and cosmic magnetogenesis.
\end{abstract}

DOI: 10.1103/PhysRevLett.126.021801

The origin of cosmic magnetic fields remains a subject of intense debate, see Refs. [1-4] for reviews. A leading hypothesis is that these fields originated in the hot and homogeneous early Universe. If this hypothesis is correct, the requirements that the magnetic fields (i) were germinated before and (ii) survived until the beginning of the structure formation epoch (when the process of their amplification started)-impose tight constraints on the possible history of the Universe, likely implying the existence of new physics $[4,5]$. This potential for serving as a bridge between the observational data and the properties of the early Universe makes both primordial magnetogenesis and magnetohydrodynamics (MHD) of ultrarelativistic plasmas research topics of fundamental importance. It has been argued that due to the weakness of nonconservation of the axial charge current in an ultrarelativistic plasma, the proper description of the evolution of primordial cosmic magnetic fields requires an extension of MHD called chiral magnetohydrodynamics [6-8], see also Refs. $[9,10]$. In chiral MHD the system of Maxwell and Navier-Stokes equations is supplemented with an extra degree of freedom-the axial chemical potential. Such an extension materially affects the predictions of the theory. In particular, chiral MHD admits for the transfer of magnetic energy from short- to long-wavelength modes of helical magnetic fields, partially compensating Ohmic dissipation in the early Universe and thus increasing

Published by the American Physical Society under the terms of the Creative Commons Attribution 4.0 International license. Further distribution of this work must maintain attribution to the author(s) and the published article's title, journal citation, and DOI. Funded by SCOAP ${ }^{3}$. their chance to survive until today [6,7,11-16]. It is worth noting that chiral MHD has drawn a lot of recent interest not only because of its importance for the description of primordial magnetic fields, but also due to its relevance to the theory of neutron stars and quark-gluon plasmas (see, e.g., Refs. [8,10-34]).

The chiral MHD description is only appropriate inasmuch as the axial current can be treated as conserved on microscopic timescales such as the momentum and energy relaxation rates. This requires the typical kinetic energy of an electron in the plasma to significantly exceed the electron mass $m_{e}$, so one can meaningfully assign chirality to each particle. In such a high-temperature regime, $T \gg m_{e}$, the axial charge decays through rare chiralityflipping processes, which are still possible due to the nonconservation of chirality introduced by a perturbatively small mass term. Surprisingly, the chirality flipping rate resulting from such processes has never been rigorously calculated [35]. The previous body of work relied on the naive estimate of the chirality flip rate

$$
\Gamma_{\text {flip }}^{\text {naive }} \propto\left(\frac{m_{e}}{T}\right)^{2} \alpha^{2} T
$$

as being second order in the small parameter responsible for chirality nonconservation $m_{e} / T$ and first order in the electron scattering rate $\Gamma_{\text {scat }} \propto \alpha^{2} T$ (see, e.g., Refs. [7,3840]), where $\alpha=e^{2} /(4 \pi)$ is the fine structure constant. This estimate is based on the simple rationale that for an ultrarelativistic particle in a definite helicity state, which up to a correction on the order of $m_{e} / T$ is the same as a definite chirality state, the helicity can only be flipped via a sideways scattering process having the rate $\Gamma_{\text {scat }}$. 


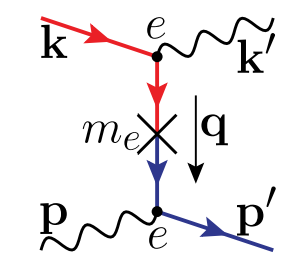

(a)

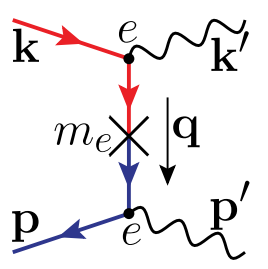

(b)

FIG. 1. The $t$-channel Compton scattering (a) and electronpositron annihilation (b) with the chirality flip in the intermediate state contributing to the chirality equilibration rate. Although naively they are of the second order in $\alpha$, their amplitudes contain infrared singularities. Regularization of these singularities leads to the result which is of the first order in $\alpha$.

The aim of the present work is to show that contrary to the naive expectation Eq. (1), the actual chirality flipping rate in an ultrarelativistic plasma is first order in $\alpha$; see Eq. (9). We focus, in particular, on the analysis of infrared singularities in the matrix elements of chirality-flipping Compton scattering and show how they effectively lead to the cancellation of one power of $\alpha$. We also briefly discuss other scattering channels which contribute to chirality flipping in the same order of perturbation theory and give the resulting leading-order asymptotic expression for the chirality flipping rate. A detailed derivation of this results in the framework of quantum field theory based linear response formalism can be found in our companion paper [41]. We note that although it is natural for kinetic coefficients associated with electron-photon scattering to be second order in the fine structure constant, there exists another known exception from this rule-the axial charge diffusion coefficient [42].

Our main idea can be summarized as follows. We consider $2 \leftrightarrow 2$ chirality flipping processes, starting from the massless QED limit and treating both the electron mass and the electron-photon coupling as perturbations (see Fig. 1). As is well known, such processes have a nonintegrable infrared singularity at small momentum transfer $[43,44]$. This signals the need for the resummation of the leading infrared divergence in all orders of the perturbation theory series. Such a resummation should generally result in an answer $\Gamma_{\text {flip }} \propto \alpha^{2} T m_{e}^{2} / q_{I \mathrm{R}}^{2}$, where $q_{I \mathrm{R}}$ is the infrared regulator scale associated with either the effective mass or the lifetime of the quasiparticle associated with the electron propagator. In a hot plasma a natural infrared scale arises from the thermal self-energy corrections to the dispersion relations of (quasi)particles. In the Supplemental Material [45], Sec. A we use hard thermal loops (HTL) resummation to show that such corrections are of the order $q_{I R} \sim \sqrt{\alpha} T$, which results in $\Gamma_{\text {flip }} \propto \alpha m_{e}^{2} / T$. We note that such an approach is not valid in the regime where the self-energy corrections are less than the electron mass. Therefore the validity range of our analysis is $T \geq m_{e} / \sqrt{\alpha} \sim 10 \mathrm{MeV}$.

Next we describe our calculations in some detail. Particle chiralities are well defined for free massless particles. Therefore we start from massless QED and treat mass as a perturbation. In plasma this means that we consider each chirality obeying its own Fermi-Dirac distribution

$$
f_{L, R}(\mathbf{k})=\frac{1}{\exp \left[\left(\epsilon_{k} \pm \mu_{5}\right) / T\right]+1} \equiv n_{F}\left(\epsilon_{k} \pm \mu_{5}\right)
$$

with chemical potentials $\pm \mu_{5}$ for right- and left-chiral particles. [For the corresponding antiparticles the chemical potentials should be taken with the opposite sign, $\left.f_{\bar{L}, \bar{R}}(\mathbf{k})=n_{F}\left(\epsilon_{k} \mp \mu_{5}\right)\right]$. The left-right chirality imbalance is then characterized by the density of axial charge

$$
q_{5}=\int \frac{d^{3} \mathbf{k}}{(2 \pi)^{3}}\left(f_{R}-f_{\bar{R}}-f_{L}+f_{\bar{L}}\right)=\frac{T^{2} \mu_{5}}{3},
$$

where in the last equality we assumed that $\mu_{5} \ll T$.

The electron mass $m_{e}$ breaks the axial symmetry and thus the axial charge relaxes to zero: $\dot{q}_{5}=-\Gamma_{\text {flip }} q_{5}$ [51]. Assuming that the chirality relaxation is the slowest equilibration process in the plasma (we give a posterior justification of the assumption of the slowness of the chirality relaxation) the thermodynamic state (2) with slowly varying $\mu_{5} \neq 0$ can still be defined. We can then use Boltzmann's kinetic theory to compute $\Gamma_{\text {flip }}$ as an asymptotic series in $m_{e} / T \ll 1$ [57].

We now proceed to the calculation of the chirality relaxation rate due to the $2 \leftrightarrow 2$ processes of Fig. 1 within the framework of Boltzmann's kinetic theory. The rate of change of the axial charge due to the $2 \leftrightarrow 2$ scattering processes is given by

$$
\dot{q}_{5}=-\int \frac{d^{3} \mathbf{k}}{(2 \pi)^{3}}\left(\mathcal{C}_{R}-\mathcal{C}_{\bar{R}}-\mathcal{C}_{L}+\mathcal{C}_{\bar{L}}\right)
$$

where

$$
\begin{aligned}
\mathcal{C}_{a}(\mathbf{k})= & \sum_{\{b c d\}} \int \frac{d^{3} \mathbf{k}^{\prime}}{(2 \pi)^{3}} \frac{d^{3} \mathbf{p}}{(2 \pi)^{3}} \frac{d^{3} \mathbf{p}^{\prime}}{(2 \pi)^{3}} \frac{\left|\mathcal{M}_{c d}^{a b}\left(k p \rightarrow k^{\prime} p^{\prime}\right)\right|^{2}}{16 \epsilon_{k} \epsilon_{k^{\prime}} \epsilon_{p} \epsilon_{p^{\prime}}}(2 \pi)^{4} \delta^{(4)}\left(k+p-k^{\prime}-p^{\prime}\right) \\
& {\left[f_{a}(\mathbf{k}) f_{b}(\mathbf{p})\left(1 \pm f_{c}\left(\mathbf{k}^{\prime}\right)\right)\left(1 \pm f_{d}\left(\mathbf{p}^{\prime}\right)\right)-\left(1 \pm f_{a}(\mathbf{k})\right)\left(1 \pm f_{b}(\mathbf{p})\right) f_{c}\left(\mathbf{k}^{\prime}\right) f_{d}\left(\mathbf{p}^{\prime}\right)\right], }
\end{aligned}
$$


is Boltzmann's collision integral. In Eq. (5), $k=\left(k^{0}, \mathbf{k}\right)$ is the 4-momentum, with $k^{0}=\epsilon_{k}=|\mathbf{k}|$ (the hard particles with $k \gtrsim T$ can be treated effectively as massless). The delta function takes into account the energy-momentum conservation in scattering. The subscripts $a, b, c, d$ run through the set of particle species $R, L, \bar{R}, \bar{L}, \gamma ; f_{a}(\mathbf{k})$ is the distribution function for the particle of type $a$ and in the expression $\pm f_{a}(\mathbf{k})$ the sign depends on the statistics of the particle $a$ (plus for a boson and minus for a fermion). The amplitudes $\mathcal{M}_{c d}^{a b}$ are found by applying Feynman's rules to the diagrams shown in Fig. 1.

Expanding the thermal Fermi-Dirac distribution functions in the collision integral on the right-hand side of Eq. (4) to the linear order in $\mu_{5}$ and using Eq. (3) we find that the chirality imbalance decays exponentially with the relaxation rate given by

$$
\begin{aligned}
\Gamma_{\text {flip }}= & \frac{3 \pi}{T^{3}} \int \frac{d^{3} \mathbf{k}}{(2 \pi)^{3}} \frac{d^{3} \mathbf{p}}{(2 \pi)^{3}} \frac{d^{3} \mathbf{q}}{(2 \pi)^{3}}\left\{n_{F}(k) n_{F}(p)\left[1+n_{B}\left(k^{\prime}\right)\right]\left[1+n_{B}\left(p^{\prime}\right)\right]\left|\mathcal{M}_{\mathrm{annih}}\right|^{2}\right. \\
& \left.+n_{F}(k) n_{B}(p)\left[1+n_{B}\left(k^{\prime}\right)\right]\left[1-n_{F}\left(p^{\prime}\right)\right]\left|\mathcal{M}_{\mathrm{Compt}}\right|^{2}\right\} \frac{\delta\left(\epsilon_{k}+\epsilon_{p}-\epsilon_{k^{\prime}}-\epsilon_{p^{\prime}}\right)}{\epsilon_{k} \epsilon_{k^{\prime}} \epsilon_{p} \epsilon_{p^{\prime}}}
\end{aligned}
$$

where $\quad \mathbf{k}^{\prime}=\mathbf{k}-\mathbf{q}, \quad \mathbf{p}^{\prime}=\mathbf{p}+\mathbf{q}, \quad$ and $\quad n_{B}(p)=$ $1 /\left[\exp \left(\epsilon_{p} / T\right)-1\right]$ is the Bose-Einstein distribution function. We note that in the weakly nonequilibrium situation $\mu_{5} \ll T$ considered here it is appropriate to take the FermiDirac distribution functions and all matrix elements in Eq. (6) at $\mu_{5}=0$.

Since we treat mass $m_{e}$ as a perturbation, we expand the matrix element of the Compton process as a perturbative series in $m_{e}$ and keep only the leading term

$$
\left|\mathcal{M}^{(1)}\right|^{2}=\frac{8 m_{e}^{2} e^{4} \epsilon_{k} \epsilon_{p}\left(1-\cos \theta_{k p}\right)}{\left(q^{2}\right)^{2}},
$$

where $\theta_{k p}$ is an angle between vectors $\mathbf{k}$ and $\mathbf{p}$. This matrix element contains a nonintegrable singularity at $q=0$ which needs to be regularized by the environmental effects. To that end, we perform a partial resummation of the perturbative expansion in $\alpha$ to take into account the thermal self-energy corrections to the dispersion relations of quasiparticles:

$$
\left|\mathcal{M}^{(1), \text { therm }}\right|^{2}=\frac{8 m_{e}^{2} e^{4} \epsilon_{k} \epsilon_{p}\left(1-\cos \theta_{k p}\right)}{\left|(q-\varpi)^{2}\right|^{2}}
$$

where $\varpi$ is a four-vector associated with the retarded selfenergy of the intermediate particle by $\varpi^{\mu}=\operatorname{tr}\left(\gamma^{\mu} \Sigma_{\text {ret }}\right) / 4$; see Refs. [58,59] and the discussion around Eq. (A.3) in the Supplemental Material [45] for more details. The presence of $\varpi$ regularizes the infrared divergence at $q \sim \sqrt{\alpha} T$.

Using the explicit expressions for the electron selfenergy in the HTL approximation, we find that the chirality flipping rate

$$
\Gamma_{\text {flip }}=C \times \alpha \frac{m_{e}^{2}}{T},
$$

where the constant $C \approx 0.24$ (see Ref. [45], Sec. A).
Next, we briefly discuss other processes that contribute to the chirality flipping rate in the same order of perturbation theory as the Compton process. One such process is shown in Fig. 2. Its contribution to the chirality flipping rate can be estimated in a way similar to the $2 \leftrightarrow 2$ case [see Eq. (6)]

$$
\begin{aligned}
\Gamma_{\text {flip }}^{1 \leftrightarrow 2} \propto & \frac{1}{T^{3}} \int d^{3} \mathbf{k} d^{3} \mathbf{p} d^{3} \mathbf{q} n_{F}\left(\epsilon_{k}\right)\left[1+n_{B}\left(\epsilon_{p}\right)\right]\left[1-n_{F}\left(\epsilon_{q}\right)\right] \\
& \frac{\left|\mathcal{M}_{k \rightarrow p q}\right|^{2}}{\epsilon_{k} \epsilon_{q} \epsilon_{p}} \delta^{(3)}(\mathbf{k}-\mathbf{q}-\mathbf{p}) \delta\left(\epsilon_{k}-\epsilon_{q}-\epsilon_{p}\right)
\end{aligned}
$$

where the matrix element reads as

$$
\left|\mathcal{M}_{k \rightarrow p q}\right|^{2}=2 e^{2} m_{e}^{2} \frac{k \cdot p}{k^{2}} .
$$

In vacuum, $\epsilon_{k}=|\mathbf{k}|$ and the process is only allowed for strictly collinear momenta of participating particles. Because of this kinematical constraint the process has an extremely unstable phase volume that can even be wiped out by an infinitesimal deformation of the dispersion curves of the particles. At the same time, the singularity of the matrix element (11) at $k=0$ leads to a nonintegrable divergence inside the available phase volume resulting in an uncertainty of $0 / 0$ type. The resolution of this

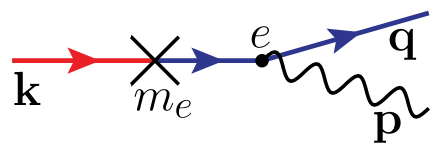

FIG. 2. One of the $1 \leftrightarrow 2$ collinear processes with chirality flip of the incoming electron (states with different chiralities are shown in different colors). Although for massless particles the process has a finite phase space, it is very sensitive to any modification of the particles' dispersion relations. This leads to an uncertainty in the probability of such a process which is addressed in Ref. [41]. 
uncertainty requires consideration of the finite lifetime of the particles involved in scattering as well as possible effects resulting from the multiple emission of soft photons [60-65]. Such an analysis lies outside the scope of the present work. In Sec. B of the Supplemental Material [45] we explain why one should expect this contribution to the chirality flipping rate to be of the same parametric order as Eq. (9).

For details, we refer the interested reader to our companion paper, Ref. [41], where we investigate the chirality flipping rate within the framework of linear response theory. The leading-order result for the chirality flipping rate derived in Ref. [41] has the form given in Eq. (9) with the coefficient $C$, which is a logarithmically varying function of $\alpha$. For $\alpha=1 / 137$ we find

$$
C \approx 1.17
$$

Thus, we find that the actual chirality flipping rate (9) is 3 orders of magnitude as high as the previously used naive estimate $\Gamma_{\text {flip }}^{\text {naive }}$ (see, e.g., Ref. [7]).

Chirality flip across cosmic times.-Our result (9) enables us to compute the electron-mass induced the chirality flipping rate in the early Universe at temperatures $T \gtrsim m_{e} / \sqrt{\alpha}$; however, at much higher temperatures one should take into account other mechanisms responsible for chirality flipping.

At temperature above the electroweak phase transition the chirality flipping rate behaves as $\Gamma_{\text {flip }}=\left(T_{R} / M_{*}\right) T$, where $M_{*}=M_{\mathrm{Pl}} /\left(1.66 \sqrt{g_{*}}\right)$ and $T_{R} \sim 80 \mathrm{TeV}[36,37]$. The responsible processes are various $2 \leftrightarrow 2$ scatterings as well as the Higgs decay. At temperatures well below the electroweak crossover, weak scatterings preserve chirality in the limit of zero masses of all fermions. They are accompanied, however, by the subleading processes where chirality flips for one of the incoming or outgoing electrons with the probability proportional to $m_{e}^{2} /\left\langle p^{2}\right\rangle$. The corresponding estimate for the reaction rate is given by

$$
\Gamma_{\text {flip }, E \mathrm{~W}} \simeq G_{F}^{2} T^{5}\left(\frac{m_{e}}{3 T}\right)^{2} .
$$

Unlike the QED case, there is no zero mass singularities because of the massive intermediate vector bosons. There is also the contribution to the chirality flipping rate due to the Higgs (inverse) decay $\left(h \leftrightarrow e_{L}^{-} e_{R}^{+}\right.$),

$$
\Gamma_{\text {flip }, H}=\frac{3 \sqrt{2}}{\pi^{5}} G_{F} T m_{e}^{2}\left(\frac{\pi m_{H}}{2 T}\right)^{5 / 2} e^{-m_{H} / T}
$$

where $m_{H}$ is the Higgs boson mass.

These results are summarized in Fig. 3, which demonstrates that at temperatures $T \lesssim 80 \mathrm{TeV} \quad \Gamma_{\text {flip }}$ always exceeds the Hubble expansion rate; that the slowest

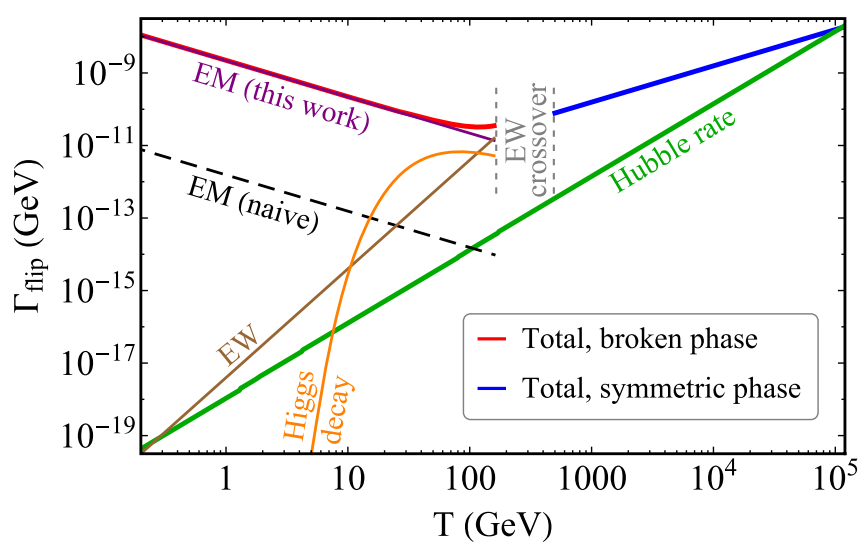

FIG. 3. Chirality flipping rates due to different processes in comparison to the Hubble expansion rate $H(T)=T^{2} / M_{*}$ as functions of temperature.

$\Gamma_{\text {flip }}(T)$ occurs at $T \simeq 100 \mathrm{GeV}$ and that below $100 \mathrm{GeV}$ the ratio $\Gamma_{\text {flip }}(T) / H(T) \gg 1$.

Conclusion and outlook.-We have shown that the chirality flipping processes for electrons in QED plasma with $T \gg m_{e}$ occur much faster than one would naively expect: it is proportional to the fine structure constant $\alpha$, rather than $\propto \alpha^{2}$ (the latter dependence holds, for example, for chirality-preserving scatterings). We used Boltzmann's collision integral to evaluate the contribution of the leadingorder $2 \leftrightarrow 2$ scattering processes (Fig. 1). As $m_{e} / T \rightarrow 0$, the matrix elements for these processes exhibit the infrared singularity. In order to obtain a meaningful result one has to proceed beyond tree-level analysis and invoke a partial resummation of the perturbation theory series. In plasma such a resummation results in the singularity being regularized not by the mass $m_{e}$ but by the thermal mass of the electron $m_{\text {th }}=e T / 2$. Our result in particular means that the chirality flipping rate is $\mathcal{O}\left(10^{3}\right)$ higher than was previously believed.

Chiral anomaly provides a coupling between the magnetic field and the axial current of electrons via the chiral magnetic effect $[6,54,66]$. Such a coupling has, in particular, been shown to lead to a special form of "inverse cascade" (transfer of the magnetic energy from smaller to larger scales) even in the absence of turbulence [6$8,11,12,15,26]$. The inverse cascade is a remarkable example of macroscopic manifestation of a microscopic quantum effect. This mechanism was, in particular, shown to increase the resilience of macroscopic magnetic fields against dissipative processes [7,15]. Chirality flipping suppresses the chiral magnetic effect, therefore it may switch off the inverse cascade before it completes the redistribution of energy between the electromagnetic modes. The present study shows that the accurate description of timescales associated with such counteracting mechanisms in a plasma requires a good microscopic understanding of the underlying quantum processes. 
Chirality flipping is not the only such mechanism. Recent microscopic simulations [67-69] hint that the anomaly induced rate of redistribution of energy between the electromagnetic modes may significantly exceed its classical estimate, presumably due to quantum effects arising at short length scales. These findings call for further revision of the MHD of axially charged plasmas based on a first-principles approach along the lines of the present study.

We are grateful to Artem Ivashko, Oleksandr Gamayun, Kyrylo Bondarenko, Alexander Monin, and Mikhail Shaposhnikov for valuable discussions. This project has received funding from the European Research Council (ERC) under the European Union's Horizon 2020 research and innovation programme (GA 694896) and from the Carlsberg Foundation. The work of O. S. was supported by the Swiss National Science Foundation Grant No. 200020B_182864.

*oleksandr.sobol@epfl.ch

[1] D. Grasso and H. R. Rubinstein, Magnetic fields in the early universe, Phys. Rep. 348, 163 (2001).

[2] K. Subramanian, Magnetic fields in the early universe, Astron. Nachr. 331, 110 (2010).

[3] A. Kandus, K. E. Kunze, and C. G. Tsagas, Primordial magnetogenesis, Phys. Rep. 505, 1 (2011).

[4] R. Durrer and A. Neronov, Cosmological magnetic fields: Their generation, evolution and observation, Astron. Astrophys. Rev. 21, 62 (2013).

[5] H. J. Hortua, Cosmological features of primordial magnetic fields, Ph.D. thesis, National University of Colombia, 2019.

[6] M. Joyce and M.E. Shaposhnikov, Primordial Magnetic Fields, Right-Handed Electrons, and the Abelian Anomaly, Phys. Rev. Lett. 79, 1193 (1997).

[7] A. Boyarsky, J. Frohlich, and O. Ruchayskiy, Self-Consistent Evolution of Magnetic Fields and Chiral Asymmetry in the Early Universe, Phys. Rev. Lett. 108, 031301 (2012).

[8] I. Rogachevskii, O. Ruchayskiy, A. Boyarsky, J. Fröhlich, N. Kleeorin, A. Brandenburg, and J. Schober Laminar and turbulent dynamos in chiral magnetohydrodynamics-I: Theory, Astrophys. J. 846, 153 (2017).

[9] M. Giovannini, Anomalous magnetohydrodynamics, Phys. Rev. D 88, 063536 (2013).

[10] L. Del Zanna and N. Bucciatini, Covariant and $3+1$ equations for dynamo-chiral general relativistic magnetohydrodynamics, Mon. Not. R. Astron. Soc. 479, 657 (2018).

[11] H. Tashiro, T. Vachaspati, and A. Vilenkin, Chiral effects and cosmic magnetic fields, Phys. Rev. D 86, 105033 (2012).

[12] Y. Hirono, D. Kharzeev, and Y. Yin, Self-similar inverse cascade of magnetic helicity driven by the chiral anomaly, Phys. Rev. D 92, 125031 (2015).

[13] M. Dvornikov and V. B. Semikoz, Influence of the turbulent motion on the chiral magnetic effect in the early Universe, Phys. Rev. D 95, 043538 (2017).

[14] E. Gorbar, I. Rudenok, I. Shovkovy, and S. Vilchinskii, Anomaly-driven inverse cascade and inhomogeneities in a magnetized chiral plasma in the early Universe, Phys. Rev. D 94, 103528 (2016).

[15] A. Brandenburg, J. Schober, I. Rogachevskii, T. Kahniashvili, A. Boyarsky, J. Fröhlich, O. Ruchayskiy, and N. Kleeorin, The turbulent chiral-magnetic cascade in the early universe, Astrophys. J. Lett. 845, L21 (2017).

[16] J. Schober, A. Brandenburg, and I. Rogachevskii, Chiral fermion asymmetry in high-energy plasma simulations, Geophys. Astrophys. Fluid Dyn. 114, 106 (2020).

[17] D.E. Kharzeev, The chiral MagnetoHydroDynamics of QCD fluid at RHIC and LHC, J. Phys. G 38, 124061 (2011).

[18] A. Boyarsky, O. Ruchayskiy, and M. Shaposhnikov, LongRange Magnetic Fields in the Ground State of the Standard Model Plasma, Phys. Rev. Lett. 109, 111602 (2012).

[19] Y. Akamatsu and N. Yamamoto, Chiral Plasma Instabilities, Phys. Rev. Lett. 111, 052002 (2013).

[20] J. M. Wagstaff and R. Banerjee, Extragalactic magnetic fields unlikely generated at the electroweak phase transition, J. Cosmol. Astropart. Phys. 01 (2016) 002.

[21] N. Yamamoto, Chiral Alfvén Wave in Anomalous Hydrodynamics, Phys. Rev. Lett. 115, 141601 (2015).

[22] A. J. Long and E. Sabancilar, Chiral charge erasure via thermal fluctuations of magnetic helicity, J. Cosmol. Astropart. Phys. 05 (2016) 029.

[23] P. Pavlović, N. Leite, and G. Sigl, Chiral magnetohydrodynamic turbulence, Phys. Rev. D 96, 023504 (2017).

[24] E. V. Gorbar, I. A. Shovkovy, S. Vilchinskii, I. Rudenok, A. Boyarsky, and O. Ruchayskiy, Anomalous Maxwell equations for inhomogeneous chiral plasma, Phys. Rev. D 93, 105028 (2016).

[25] S. Sen and N. Yamamoto, Chiral Shock Waves, Phys. Rev. Lett. 118, 181601 (2017).

[26] J. Schober, I. Rogachevskii, A. Brandenburg, A. Boyarsky, J. Fröhlich, O. Ruchayskiy, and N. Kleeorin, Laminar and turbulent dynamos in chiral magnetohydrodynamics. II. Simulations, Astrophys. J. 858, 124 (2018).

[27] Y. Hirono, D. E. Kharzeev, and Y. Yin, New quantum effects in relativistic magnetohydrodynamics, Nucl. Phys. A967, 840 (2017).

[28] K. Hattori, Y. Hirono, H.-U. Yee, and Y. Yin, MagnetoHydrodynamics with chiral anomaly: Phases of collective excitations and instabilities, Phys. Rev. D 100, 065023 (2019)

[29] E. Gorbar, D. Rybalka, and I. Shovkovy, Second-order dissipative hydrodynamics for plasma with chiral asymmetry and vorticity, Phys. Rev. D 95, 096010 (2017).

[30] J. Schober, A. Brandenburg, I. Rogachevskii, and N. Kleeorin, Energetics of turbulence generated by chiral MHD dynamos, Geophys. Astrophys. Fluid Dyn. 113, 107 (2019).

[31] M. Dvornikov and V. B. Semikoz, Magnetic helicity evolution in a neutron star accounting for the Adler-Bell-Jackiw anomaly, J. Cosmol. Astropart. Phys. 08 (2018) 021.

[32] Y. Masada, K. Kotake, T. Takiwaki, and N. Yamamoto, Chiral magnetohydrodynamic turbulence in core-collapse supernovae, Phys. Rev. D 98, 083018 (2018).

[33] M. Mace, N. Mueller, S. Schlichting, and S. Sharma, Chiral Instabilities and the Onset of Chiral Turbulence in QED Plasmas, Phys. Rev. Lett. 124, 191604 (2020). 
[34] J. Schober, T. Fujita, and R. Durrer, Generation of chiral asymmetry via helical magnetic fields, Phys. Rev. D 101, 103028 (2020).

[35] A similar quantity, equilibration rate of right-handed electrons was computed at temperatures where electroweak symmetry is unbroken, fermions are massless, and the chirality flipping proceeds via electron-electron-Higgs Yukawa interaction [36,37]. Our computation is conceptually different, as we will explain.

[36] B. A. Campbell, S. Davidson, J. R. Ellis, and K. A. Olive, On the baryon, lepton flavor and right-handed electron asymmetries of the universe, Phys. Lett. B 297, 118 (1992).

[37] D. Bödeker and D. Schröder, Equilibration of right-handed electrons, J. Cosmol. Astropart. Phys. 05 (2019) 010.

[38] D. Grabowska, D. B. Kaplan, and S. Reddy, Role of the electron mass in damping chiral plasma instability in supernovae and neutron stars, Phys. Rev. D 91, 085035 (2015).

[39] C. Manuel and J. M. Torres-Rincon, Dynamical evolution of the chiral magnetic effect: Applications to the quark-gluon plasma, Phys. Rev. D 92, 074018 (2015).

[40] P. Pavlović, N. Leite, and G. Sigl, Modified magnetohydrodynamics around the electroweak transition, J. Cosmol. Astropart. Phys. 06 (2016) 044.

[41] A. Boyarsky, V. Cheianov, O. Ruchayskiy, and O. Sobol, companion paper, Equilibration of the chiral asymmetry due to finite electron mass in the electron-positron plasma, Phys. Rev. D 103, 013003 (2021).

[42] D.-f. Hou and S. Lin, Fluctuation and dissipation of axial charge from massive quarks, Phys. Rev. D 98, 054014 (2018).

[43] T. D. Lee and M. Nauenberg, Degenerate systems and mass singularities, Phys. Rev. 133, B1549 (1964).

[44] A. D. Dolgov and V. I. Zakharov, On conservation of the axial current in massless electrodynamics, Nucl. Phys. B27, 525 (1971).

[45] See Supplemental Material at http://link.aps.org/ supplemental/10.1103/PhysRevLett.126.021801 for additional details on calculation of the Boltzmann's collision integral for Compton scattering and annihilation processes, on the electron self-energy resummation in HTL approximation, and on the contribution to the chirality flipping rate from $1 \leftrightarrow 2$ processes, which includes Ref. [46-50].

[46] M. Le Bellac, Thermal Field Theory, Cambridge Monographs on Mathematical Physics (Cambridge University Press, Cambridge, England, 1996).

[47] J. Ghiglieri, A. Kurkela, M. Strickland, and A. Vuorinen, Perturbative thermal QCD: Formalism and applications, Phys. Rep. 880, 1 (2020).

[48] M. H. Thoma, Applications of high temperature field theory to heavy ion collisions, in Quark-Gluon Plasma 2 (World Scientific, Singapore, 1995).

[49] J.-P. Blaizot and E. Iancu, Lifetimes of quasiparticles and collective excitations in hot QED plasmas, Phys. Rev. D 55, 973 (1997).

[50] J.-P. Blaizot and E. Iancu, Lifetime of Quasiparticles in Hot QED Plasmas, Phys. Rev. Lett. 76, 3080 (1996).

[51] Apart from chirality flips, conservation of the axial charge is also violated due to the chiral anomaly $[52,53]$. The effect of the anomaly can be rigorously distinguished from chirality flipping collisions due to the existence of a global charge whose conservation is respected by the former, however, is violated by the latter [54-56]. Here we focus on the contribution of genuine chirality flipping processes only.

[52] S. L. Adler, Axial vector vertex in spinor electrodynamics, Phys. Rev. 177, 2426 (1969).

[53] J. S. Bell and R. Jackiw, A PCAC puzzle: $\pi^{0} \rightarrow \gamma \gamma$ in the $\sigma$ model, Nuovo Cimento A 60, 47 (1969).

[54] A. Y. Alekseev, V. V. Cheianov, and J. Frohlich, Universality of Transport Properties in Equilibrium, Goldstone Theorem and Chiral Anomaly, Phys. Rev. Lett. 81, 3503 (1998).

[55] J. Fröhlich and B. Pedrini, New applications of the chiral anomaly, in International Conference on Mathematical Physics 2000, Imperial College (London), edited by A. S. Fokas, A. Grigoryan, T. Kibble, and B. Zegarlinski (World Scientific, Singapore, 2000).

[56] J. Fröhlich and B. Pedrini, Axions, quantum mechanical pumping, and primeval magnetic fields, in Statistical Field Theory, edited by A. Cappelli and G. Mussardo (Kluwer, Amsterdam, 2002).

[57] Mass is a relevant operator that changes the dispersion relation (chiral fermion has 2 degrees of freedom, while massive Dirac fermion has 4). This makes the computation conceptually different from Refs. [36,37] where the rate was determined by the Yukawa interaction (marginal operator).

[58] J.-P. Blaizot and E. Iancu, A Boltzmann equation for the QCD plasma, Nucl. Phys. B557, 183 (1999).

[59] J.-P. Blaizot and E. Iancu, The quark gluon plasma: Collective dynamics and hard thermal loops, Phys. Rep. 359, 355 (2002).

[60] R. Baier, D. Schiff, and B. Zakharov, Energy loss in perturbative QCD, Annu. Rev. Nucl. Part. Sci. 50, 37 (2000).

[61] A. Kovner and U.A. Wiedemann, Gluon radiation and parton energy loss, in QuarkGluon Plasma 3 (World Scientific, Singapore, 2003), pp. 192-248.

[62] P. Aurenche, F. Gelis, and H. Zaraket, Landau-Pomeranchuk-Migdal effect in thermal field theory, Phys. Rev. D 62 , 096012 (2000).

[63] P. B. Arnold, G. D. Moore, and L. G. Yaffe, Photon emission from ultrarelativistic plasmas, J. High Energy Phys. 11 (2001) 057.

[64] P. B. Arnold, G. D. Moore, and L. G. Yaffe, Photon and gluon emission in relativistic plasmas, J. High Energy Phys. 06 (2002) 030.

[65] P. B. Arnold, G. D. Moore, and L. G. Yaffe, Effective kinetic theory for high temperature gauge theories, J. High Energy Phys. 01 (2003) 030.

[66] A. Vilenkin, Equilibrium parity violating current in a magnetic field, Phys. Rev. D 22, 3080 (1980).

[67] D. G. Figueroa and M. Shaposhnikov, Lattice implementation of Abelian gauge theories with Chern-Simons number and an axion field, Nucl. Phys. B926, 544 (2018).

[68] D. G. Figueroa and M. Shaposhnikov, Anomalous nonconservation of fermion/chiral number in Abelian gauge theories at finite temperature, J. High Energy Phys. 04 (2018) 026.

[69] D. G. Figueroa, A. Florio, and M. Shaposhnikov, Chiral charge dynamics in Abelian gauge theories at finite temperature, J. High Energy Phys. 10 (2019) 142. 